\section{POS0301 NO MAJOR DIFFERENCES BETWEEN PATIENTS WITH CHRONIC INFLAMMATORY RHEUMATIC DISEASE WHO UNDERWENT MONO- OR MULTISWITCHING OF BIOSIMILARS IN ROUTINE CARE (PERCEPTION STUDY)}

S. Gall ${ }^{1}$, U. Kiltz ${ }^{1}$, T. Kobylinski ${ }^{1}$, I. Andreica ${ }^{1}$, K. Vaupel $^{1}$, X. Baraliakos ${ }^{1}$, J. Braun ${ }^{1} .^{1}$ Rheumazentrum Ruhrgebiet at Ruhr-University Bochum, Rheumatology, Herne, Germany

Background: The increasing availability of biosimilars (bsDMARDs) has created a financial incentive to encourage switching to cheaper products ("non-medical switch") leading to different switching scenarios. While the clinical efficacy and safety of multiswitching seems to be established (1), limited data on patients' knowledge about bsDMARDs and satisfaction with care are available.

Objectives: The aim of our study was to learn more about the outcome of monoand multiswitching scenarios in routine care with respect to patients' attitudes towards bsDMARDs in chronic inflammatory rheumatic diseases (CIRD) such as rheumatoid arthritis (RA), axial spondyloarthritis $(\operatorname{axSpA})$ and psoriatic arthritis (PsA).

Methods: Consecutive patients with CIRD who were planned to switch treatment of one adalimumab biosimilar (ADA-bsDMARD) to another ADA-bsDMARD were recruited. The number of previous ADA-bsDMARD categorized the patients into: monoswitch $=1$ and multiswitch $=>1$. Demographics and standard assessments using validated outcome parameters for disease activity, physical function, and patient satisfaction with care (Leeds Satisfaction Questionnaire (LSQ) were documented. LSQ contains items on five subscales (provision of information; empathy with the patient; attitude to the patient; access to and continuity with the care giver; and technical competence) and a general satisfaction scale. Knowledge about bsDMARDs was recorded using a structured questionnaire.

Results: Out of 90 patients in total, there were 42 with a monoswitch and 48 with a multiswitch scenario (Table 1). Patients were satisfied with care irrespective of the switching scenario. However, the knowledge about bsDMARDs was generally rather low (Figure 1). Less than one third of patients was able to identify correct answers about manufacturing, efficacy/safety issues, approval status and costs of bsDMARDs. However, when comparing the two switch scenarios, no differences in disease characteristics nor in satisfaction with care were found. Also the number of switches had not increased the knowledge about bsDMARDs.

Conclusion: This study shows that multiswitching did not lead to reduced satisfaction with care in patients on treatment with bsDMARDs. Especially, the number of switches did have no negative impact on patients satisfaction. The observation that patients who underwent multiple switches had no more knowledge about bsDMARDs than patients who just had one switch may just be explained by the positive experience most patients had with switching.

REFERENCES:

[1] Kiltz U et al. Ann Rheum Dis 2020;79 (supplement 1):1872

Table 1. Patients and disease characteristics stratified by switch scenario

\begin{tabular}{llll}
\hline Variables $^{*}$ & Monoswitch $(\mathbf{n}=\mathbf{4 2})$ & Multiswitch $(\mathbf{n}=\mathbf{4 8})$ & P-Wert \\
\hline Sex, male, $\mathrm{n}(\%)$ & $23(54.7)$ & $26(54.2)$ & \\
Age, years & $44(14)$ & $51(11)$ & \\
Rheumatoid Arthritis, $\mathrm{n}(\%)$ & $14(33.3)$ & $7(14.6)$ & \\
Axial Spondyloarthritis, $\mathrm{n}(\%)$ & $23(54.8)$ & $31(64.6)$ & \\
Psoriatic arthritis, $\mathrm{n}(\%)$ & $5(11.9)$ & $10(20.8)$ & \\
Disease duration, years & $9.2(2.5)$ & $10.6(6.7)$ & 0.48 \\
DAS28 & $2.2(1.2)$ & $2.9(0.7)$ & 0.13 \\
HAQ & $1.2(0.6)$ & $1.2(0.5)$ & 0.91 \\
ASDAS & $2.1(1.2)$ & $1.6(1)$ & 0.70 \\
BASFI & $4.6(2.9)$ & $3.7(2.9)$ & 0.87 \\
Patient satisfaction & & & \\
LSQ-General (1-5) \# & $3.7(0.7)$ & $3.9(0.6)$ & 0.58 \\
LSQ-Information (1-5) & $3.7(0.6)$ & $3.6(0.4)$ & 0.20 \\
LSQ-Empathy (1-5) & $3.6(0.6)$ & $3.5(0.5)$ & 0.57 \\
LSQ-Technical (1-5) & $4.1(0.5)$ & $4.1(0.5)$ & 0.51 \\
LSQ-Attitude (1-5) & $3.8(0.7)$ & $3.9(0.5)$ & 0.62 \\
LSQ-Access (1-5) & $3.7(0.6)$ & $3.8(0.6)$ & 0.70 \\
\hline
\end{tabular}

*values in mean $(\mathrm{SD}) \#$ values of 1 indicate dissatisfaction

Disclosure of Interests: None declared

DOI: 10.1136/annrheumdis-2021-eular.1742
Knowledge about bsDMARDS

$$
\text { multiswitch =Monoswitch }
$$

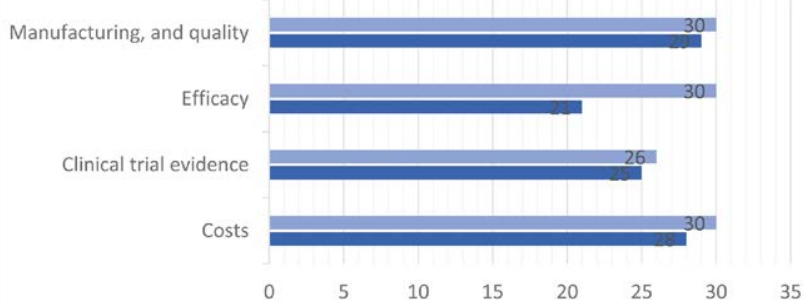

Figure 1. Knowledge about biosimilarsbsDMARDs: biosimilar disease-modifying anti-rheumatic drugs

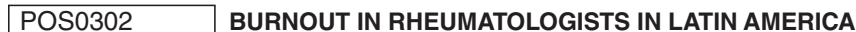

M. Intriago ${ }^{1}$, G. Maldonado ${ }^{2}$, R. Guerrero ${ }^{1}$, E. Soriano ${ }^{3}$, L. Moreno ${ }^{4}$, C. Rios ${ }^{1,4}$. ${ }^{1}$ Universidad Espiritu Santo, Rheumatology, Samborondon, Ecuador; ${ }^{2}$ Loyola MacNeal Hospital, Internal Medicine, Berwyn, United States of America; ${ }^{3}$ Hospital Italiano, Rheumatology, Buenos Aires, Argentina; ${ }^{4}$ Centro de Reumatología y Rehabilitación, Rheumatology, Guayaquil, Ecuador

Background: Rheumatology is considered a low-risk specialty, but studies have shown a prevalence of burnout between $42 \%$ to $51 \%^{1,2}$. Factors associated with it include the use of electronic medical records, administrative tasks and prolonged working hours. This has several repercussions such as higher prevalence of depression/anxiety and less productivity.

Objectives: To determine the prevalence of burnout in rheumatologists in Latin America and the factors associated with it.

Methods: Cross-sectional study based on a survey completed through Google Forms platform that was sent by the national rheumatology associations of Latin America. Burnout was assessed with the Maslach Burnout Inventory (MBI) with cut off values of $\geq 27$ for emotional exhaustion (EE), $\geq 10$ for depersonalization (DP), and $\leq 33$ for personal accomplishment (PA). Other variables were studied such as demographics, working conditions, satisfaction, comorbidities, depression using PHQ-9 and happiness using the Subjective Happiness Scale. Data was analyzed using the statistical program SPSS v.22.

Results: 297 rheumatologists from 15 countries were included, mainly Argentina $(28.3 \%)$, Brazil $(26.3 \%)$ and Mexico $(12.8 \%)$. The majority were women $62 \%$, $42.4 \%$ worked in public hospitals with an average of $40.1 \pm 14.2$ hours per week. $31.3 \%$ did research, $13.1 \%$ clinical trials, $56.6 \%$ teaching, and $42.8 \%$ administrative work. $36 \%$ received an annual income less than $25 \mathrm{~K}$.

$56.6 \%$ had burnout in at least 1 dimension. According to the dimensions, $35.7 \%$ had burnout in EE, $25.6 \%$ in PA and $26.6 \%$ in DP. $32.3 \%$ had burnout in only 1 dimension, $17.2 \%$ in two and $7.1 \%$ in all three dimensions. Only $20.2 \%$ thought they had burnout, $9.1 \%$ were currently with professional help and $15.8 \%$ had sought help in the past. $72.1 \%$ said they were willing to participate in a program to reduce burnout.

The rheumatologists with burnout were younger than those without burnout (46.5 vs 49.9 years, $p=0.015$ ), mentioned more frequently that they would like to decrease the number of working hours $(56.5 \%$ vs $36.4 \%, p=0.002)$ and had lower practice satisfaction (5.2 vs $6.2, p<0.001)$ and income satisfaction (3.4 vs $4.6, p<0.001)$. Those with burnout were less happy than those without burnout (5.1 vs $5.9, p<0.001$ ) and had higher PHQ-9 (7.7 vs $2.5, p<0.001)$. In the burnout group, there was a higher percentage with an income less than $\$ 25 \mathrm{~K}$ year $(45.7 \%$ vs $25.4 \%, p=0.008)$, presence of comorbidities $(53.6 \%$ vs $40.3 \%$, $\mathrm{p}=0.023)$, anxiety ( $11.9 \%$ vs. $2.3 \%, p=0.002)$, use of SSRIs/SNRIs (19\% vs $8.5 \%, p=0.011)$, suicidal thoughts ( $13.6 \%$ vs $1.6 \%, p<0.001)$ and low self-esteem $(12.5 \%$ vs $3.1 \%, p<0.001)$. There was a higher percentage of rheumatologists involved in teaching in the group without burnout $(66.7 \%$ vs $48.8 \%, p=0.002)$. No statistically significant associations were found between burnout and sex, exercise, alcohol consumption, duration of career, main workplace, type of clinical practice, duration of vacation, clinical research, clinical trials and administrative work.

Conclusion: Burnout affects near half of rheumatologists in Latin America and was associated to younger age, long working hours, low satisfaction, less happiness, higher PHQ-9, suicidal thoughts, anxiety, income, presence of comorbidities and low self-esteem. Most of the rheumatologists were willing to participate in programs to reduce burnout which represents an opportunity for interventions to reduce this syndrome. 